\title{
Wenn Mitarbeiter zu Botschaftern werden
}

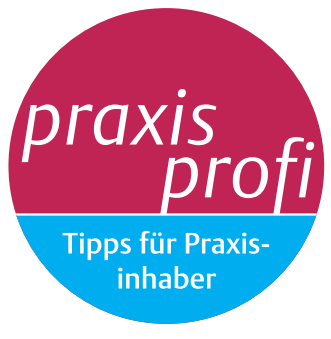

\section{Corporate-Influencer Ein Blick über den Tellerrand der bisherigen, traditionellen Marketing-}

strategien lohnt sich auch für Therapieeinrichtungen: Mithilfe von Corporate-Influencern

können sie sowohl Patienten als auch potenzielle neue Mitarbeiter ansprechen.

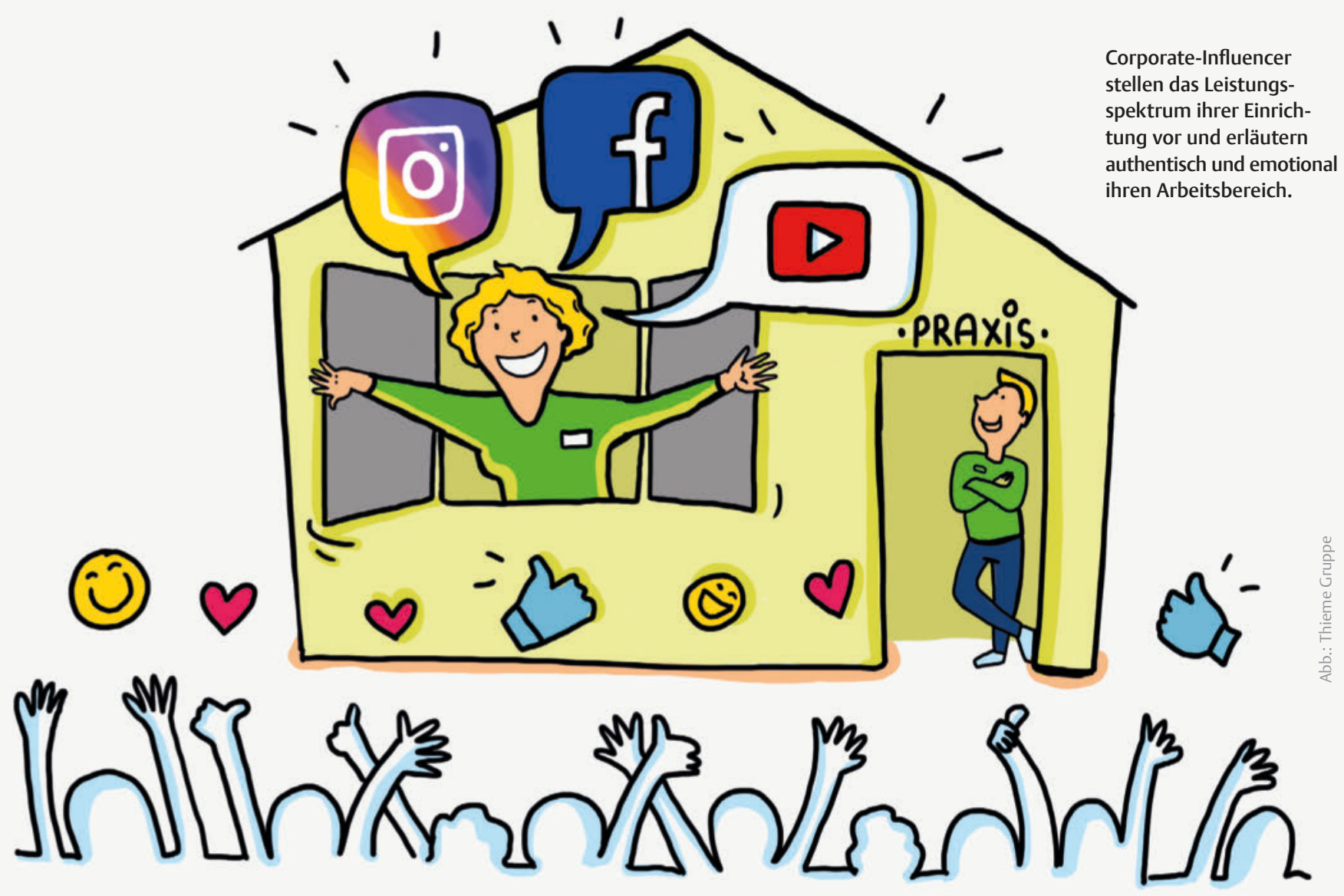

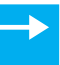

Dr. Johannes - unter diesem Namen betreibt der Arzt Dr. Johannes Wimmer seinen YouTube-Kanal. Mit über 1,6 Mio. Aufrufen und mehr als 13.000 Abonnenten gibt er nach eigener Aussage Antworten auf jede medizinische Frage und Einblicke in den Arztalltag. Er begann seine Nebenkarriere als
Influencer in der wöchentlich im NDR ausgestrahlten Sendung „Visite“ und ist zudem Buchautor. Bis heute hatte er zahlreiche Fernsehauftritte, beispielsweise 2016 bei Markus Lanz. Der charismatische Arzt, der auf Facebook, Twitter und YouTube aktiv ist, stellte also 2016 für seinen damaligen Arbeitgeber, das UKE Hamburg, den perfekten CorporateInfluencer dar. Und das im klassischen Sinne: Der Botschafter ist per se schon bekannt und hat auf seinen diversen Social-Media-Kanälen eine Reichweite und Authentizität, die für das Klinikum in diesem Fall interessant war. Das UKE durfte sich über einen positiven Image- 
transfer freuen. Und auch der Fachbereich der Dermatologie, in dem Dr. Wimmer dort tätig war, hat durch ihn und seine Reichweite on- und offline, sprich in seinen Social-MediaKanälen und bei Fernsehauftritten und Vorträgen, an Bekanntheit gewonnen.

\section{Healthcare Influencer aus den eigenen}

Reihen $\rightarrow$ Früher war das Gesicht nach außen oft der leitende Therapeut oder Praxisinhaber. Heute kann er das schon aus zeitlichen Gründen gar nicht mehr alleine leisten. Denn
Corporate-Influencern die traditionellen Tools des Marketings zu ergänzen.

Gerade im Gesundheitswesen lässt sich das Thema Glaubwürdigkeit jedoch am besten mit Influencern besetzen, die einen Bezug zur Praxis haben. Warum also nicht einen Influencer aus den eigenen Reihen aufbauen oder sich zunutze machen? Schließlich haben diese im besten Falle eine hohe Identität mit der Praxis. Die Mitarbeiterzufriedenheit sollte dabei gewährleistet sein, denn nur durch Authentizität kann man eine positive Resonanz erwarten.

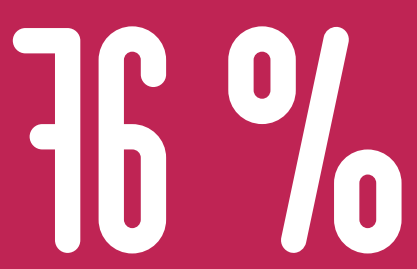

der Pressesprecher in Deutschland sind der Meinung, dass InfluencerPR an Bedeutung gewinnt. Dennoch gehört dieser Bereich noch lange nicht zum Tagesgeschäft, nur $40 \%$ der PR-Profis setzen in der eigenen Arbeit bislang auf Influencer.

PR-Trendmonitor

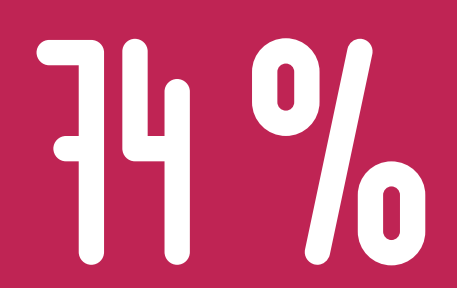

der deutschen Internetnutzer sind auf YouTube aktiv. Facebook belegt nur noch Platz drei (69\%) und liegt damit noch hinter WhatsApp (71\%). Social Media Atlas 2019

welcher Therapeut in einer Führungsposition bedient mit Leichtigkeit Facebook, Twitter, Instagram, schreibt einen Blog und broadcastet gekonnt mittels Facebook live - und das neben seinem eigentlichen Tagesgeschäft? Der Tag hat schließlich für jeden nur 24 Stunden. Daher kann es sinnvoll sein, mit

\section{Via Facebook \& Co. Fachkräfte gewinnen}

$\rightarrow$ Ein Beispiel für die Nutzung sozialer Netzwerke zu Marketingzwecken ist die KarriereFanpage der K\&S-Seniorenresidenz auf Facebook. Vorreiter auf anderen Web-Kanälen wie YouTube sind die Kursana-Residenzen, die mit einem emotionalen Recruitingfilm (YouTube:
Kursana.mp4) zum einen das Unternehmen präsentieren, zum anderen eine Tür, nicht nur zu potenziellen Bewerbern, öffnen.

Ebenso wagen sich Krankenhäuser bereits auf digitales Terrain, wie man am Blog des Rotkreuzklinikums München sehen kann. Das Klinikum Dortmund gGmbH geht mit seiner Ausbildungsoffensive \#GameChanger und seinem Imagefilm neue Wege. So auch das Universitätsklinikum Hamburg-Eppendorf mit der Plattform „jukebox“. In allen genannten Fällen übernehmen Klinikmitarbeiter die Rolle der Corporate-Influencer, stellen Teile des Leistungsspektrums ihrer Arbeitsstätten vor, zeigen, was sie ausmacht und wie sie arbeiten, und erläutern authentisch und emotional ihren Arbeitsbereich und ihre Aufgaben.

Hierfür ist es aber immens wichtig, sowohl im Binnen- als auch im Außenauftritt konsistent eine Arbeitgebermarke zu leben. Das nach außen getragene Bild sollte daher auch der Innenansicht neuer Arbeitnehmer standhalten - sonst sind diese schnell wieder weg. Hat hier ein Umdenken stattgefunden, ist der erste Schritt, zu analysieren, über welche Kanäle die „richtigen“ Bewerber zur Einrichtung finden. Denn es reicht nicht, einfach eine Stellenanzeige auch noch bei Facebook einzustellen.

\section{Influencer in Therapieeinrichtungen $\rightarrow$}

Viele Therapiepraxen haben sich spezialisiert. Um ihre Schwerpunkte nach außen zu kommunizieren, können sie neben traditionellen Marketingstrategien auch auf soziale Netzwerke setzen. Das macht die Einrichtung sowohl bei Patienten als auch in der Fachwelt bekannt. Für die Aufgabe geeignet sind Mitarbeiter, die Freude daran haben, ihren Arbeitsalltag auf sozialen Netzwerken zu teilen. Zum Beispiel berichten sie von aktuellen Fortbildungen, posten Bilder vom gemeinsamen Teamausflug und stellen die neuen Praxisräume vor. Denkbar wäre es auch, Erfolgsgeschichten zu präsentieren, sofern der jeweilige Patient einverstanden ist. Wen würde es nicht berühren, ein Foto von einer Patientin zu sehen, die trotz ihrer Hemiplegie mit ihrem Partner tanzt, oder einen Boulderer zu bestaunen, der nach einem knöchernen Sehnenausriss das erste Mal wieder am Felsen hängt.

Vertrauen ist das $\mathbf{A}$ und $\mathbf{0} \rightarrow$ Sagt Ihnen Magdalena Rogl etwas? Sie ist die wohl erfolgreichste Markenbotschafterin hierzulande und 


\section{Perspektiven | Marketingstrategie}

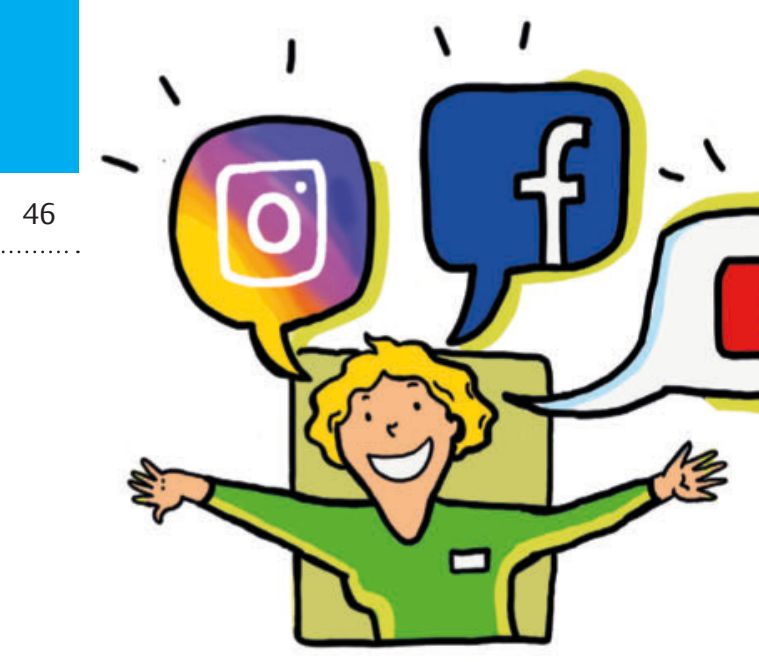

$\rightarrow$ Eigenschaften

\section{Das sollte ein Markenbotschafter mitbringen}

\author{
Engagement \\ Auch außerhalb der normalen \\ Arbeitszeiten

\begin{abstract}
Authentizität und Nahbarkeit Er spricht in seiner Sprache und bespielt seine Kanäle nur in geringen Dosen mit Arbeitsthemen. Ansonsten steht er mit beiden Beinen im Leben.
\end{abstract}

Lust und Talent für Kommunikation Ohne geht's nicht.

\section{Kompetenz}

Er treibt seine Themen aus der fachlichen Expertise heraus und ist somit glaubwürdig und relevant für seine Zielgruppe.

\section{Vernetzung und Reichweite Im besten Falle. Manchmal geht es aber auch ohne.}

\section{Begeisterung für Social Media} Dabei sollte er nur auf Plattformen eingesetzt werden, auf denen er aktiv ist bzw. die zu seinem Typ und seinen Themen passen.

\section{Offenheit}

Grundsätzlich sollte er sich wohlfühlen, wenn er unter Menschen ist und zum Beispiel auf Bühnen im Mittelpunkt steht.

das Gesicht von Microsoft Deutschland. Und sie findet, dass prinzipiell jeder Influencer werden kann. In einem Interview mit dem Magazin „PR Report“ weist sie aber darauf hin, dass ein Corporate-Influencer nur dann interessant und erfolgreich sei, wenn er mit der persönlichen Meinung nicht hinterm Berg halte und dem Leser das Gefühl gebe, einen Blick hinter die Kulissen werfen zu dürfen. Das setze jedoch einiges an Vertrauen auf beiden Seiten voraus: in die Mitarbeiter und umgekehrt ins Unternehmen. Dennoch sollten Voraussetzungen für Corporate-Influencer geschaffen werden, zum Beispiel durch Unternehmens-Guidelines, die Sicherheit vermitteln. Denn: Jeder muss wissen, woran er ist, wenn er in seinen Social-Media-Kanälen auch für seinen Arbeitgeber unterwegs ist. Dafür ist es wichtig, dass sich der leitende Therapeut oder der Praxisinhaber klar positioniert am besten mit positivem Beispiel vorangeht und eine Vorbildrolle einnimmt, indem er online sichtbar ist.

D.

Der Influencer sollte Geschichten erzählen, die emotional berühren.

$\rightarrow$ Planung ist die halbe Miete, in diesem Fall sogar die ganze: Mal schnell eine Aktion mit einem Corporate-Influencer zu realisieren, ergibt keinen Sinn, wenn man strategische Ziele verfolgt. Erst die Theorie, dann die Praxis. Oder es besser lassen.

$\rightarrow$ Reichweite, Reichweite, Reichweite: Sie ist sicherlich wichtig und gerade im klassischen Sinn ausschlaggebend bei der Entscheidung für einen Corporate-Influencer. Aber es geht auch anders. Follower, Abonnenten und Likes sind kein Garant für einen Erfolg; das Storytelling ist wichtig. Der Influencer sollte Geschichten erzählen, die die Zielgruppe emotional berühren und authentisch informieren, beispielsweise Erfolgsgeschichten aus dem Therapiealltag $\rightarrow$ Content-Mix: Corporate Influencer sollten der Öffentlichkeit neben schönen Geschichten auch konkrete Informationen anbieten. Dazu könnten sie über innovative Projekte, interne Fortbildungen oder Arbeitsmethoden berichten.

Was Einrichtungen beachten sollten $\rightarrow$ Folgende Punkte können hilfreich für den Einsatz von Corporate-Influencern in Therapieeinrichtungen sein.

$\rightarrow$ Aus den eigenen Reihen rekrutieren: Corporate-Influencer wie Patienten oder Mitarbeiter wirken glaubwürdiger als „gekaufte Influencer“, die ihre Dienste anbieten. Man fährt besser mit Influencern, die dies in erster Linie aus Überzeugung und intrinsischen Beweggründen tun.

$\rightarrow$ Am Anfang steht die gründliche Recherche: Influencer geben viel über sich preis. Sie nutzen ihre privaten Netzwerke, um ab und an auch mal ein Jobthema zu posten. Daher sollte man unbedingt überprüfen, ob die Zielgruppe der Follower des Influencers auch die ist, die man erreichen will.
Fazit $\rightarrow$ Corporate-Influencer sind spannende Persönlichkeiten. Sie tragen ihre Botschaft nach außen. Sie können eine sinnvolle Ergänzung des Marketings sein, da sie einen neuen, authentischen Zugang zu den Inhalten einer Therapieeinrichtung ermöglichen. Aber: Ein gewisser Kontrollverlust seitens der Einrichtung ist unverzichtbar, um die Authentizität der Botschafter zu bewahren. Darüber hinaus sollten sich Corporate-Influencer sowohl an Vorbildern aus der Führungsebene orientieren können als auch einen festen Ansprechpartner und Social-Media-Guidelines haben, die den groben Rahmen feststecken. Dann kann der Einsatz von Corporate-Influencern zu einer Win-win-Situation für beide Seiten werden.

Alexandra Heeser 